\title{
Effect of motor cognition program for improving temporal-spatial timing memory ability with mild cognitive impairment patients
}

\author{
Sooyeon Kim* \\ Department of Sports Medicine, Cha University, Pocheon, Korea
}

This exploratory study evaluated motor cognition program for improving temporal-spatial timing memory ability with mild cognitive impairment (MCI) patients. The purpose was to explore the efficacies of motor cognition program according to practice methods, centering on coordination and observation pattern. Two practice methods were applied to the $40 \mathrm{MCl}$ elder. In experiment 1, participants divided into two group as, one-hand practice group $(n=20)$ and both-hands practice group $(n=20)$. In experiment 2, participants divided into two group as, active observation group $(n=20)$ and passive observation group $(n=20)$. The participant was asked to alternatively press two buttons 6 times with the index finger hand with goal rhythm pattern $(3,600 \mathrm{msec}$ in total duration). In coordination pattern, bimanual practice was more effective for improving temporal-spatial timing memory ability than unilateral practice. In observation pattern, active observation showed better learning effect than passive observation. However, there was a learning effect even in passive observation pattern. Such a result claimed for the elderly, who has problem to do daily activity, could use observation of temporal-spatial timing task for improving cognitive ability.

Keywords: Mild cognitive impairment, Motor cognition program, Temporal-spatial memory ability

\section{INTRODUCTION}

The growth of the elderly population and the number of people suffering from dementia has been globing problem to affects negative economic impact. To help dementia population to live independently for as long as possible is a way to contain these costly effects. Dementia is coupled with a progressive decline of perception \& cognition, motor ability, and memory functions (Dinse et al., 2009). The effects of cognitive training for the development and prevention of cognitive abilities in mild cognitive impairment (MCI), a transitional stage between normal brain condition and dementia, has frequently been shown during recent decades (Rapp et al., 2002; Fernandez-Ballesteros et al., 2003). More recently, many studies for the MCI have shown that improving motor ability through physical exercise programs has beneficial effects on cognitive performance (Heyn et al., 2004).

Many studies employ physical exercise programs as a method to enhance cognitive performance.

There are few studies addressing motor cognition program to develop cognitive ability in rehabilitation field (Summers et al., 2007), while there are many studies focusing motor cognition program to develop cognitive ability for the normal population. Temporal-spatial timing memory ability according to sensory information condition has been examined, centering on perception-motion relationship (Maeda et al., 2004). However, it is hard to find a study about motor learning strategies, focusing motor cognition program for improving temporal-spatial timing memory ability in rehabilitation field.

This exploratory study evaluated motor cognition program for improving temporal-spatial timing memory ability with MCI pa-
${ }^{*}$ Corresponding author: Sooyeon Kim (iD https://orcid.org/0000-0003-3704-1597 Department of Sports Medicine, Cha University, 120 Haeryong-ro, Pocheon 11160, Korea

Tel \& Fax: +82-31-782-0683, E-mail: sooyeonkim76@gmail.com

Received: July 23, 2017 / Accepted: August 23, 2017
This is an Open Access article distributed under the terms of the Creative Commons Attribution Non-Commercial License (http://creativecommons.org/licenses/by-nc/4.0/) which permits unrestricted non-commercial use, distribution, and reproduction in any medium, provided the original work is properly cited. 
tients. The purpose was to explore the efficacies of motor cognition program according to practice methods, centering on coordination and observation pattern.

\section{MATERIALS AND METHODS}

\section{Participants}

For this study, 40 participants were identified and recruited voluntarily at the Seoul S center for dementia. They underwent an evaluation by a neurologist and completed a Korean version of Mini-Mental Status Examination. Forty participants were divided into two groups-one-hand practice group $(\mathrm{OHG} ; \mathrm{n}=20)$ and both-hands practice group (BHG; $\mathrm{n}=20$ ).

\section{Apparatus and task}

As the experiment apparatus, a personal computer with a color monitor, a standard keyboard and a speaker is used (Fig. 1). A customized computer program, Rhythm Perception and Timing Coordination program designed for this experiment, controlled all the experimental procedures on the computer monitor, provided feedback to the participants, and collected data for further analysis. Participants were asked to respond as fast and accurate as possible once they perceived the sequence by pressing one response key on unilateral trials and both response keys on bilateral trials, responding the goal pattern presented with visual-auditory stim-

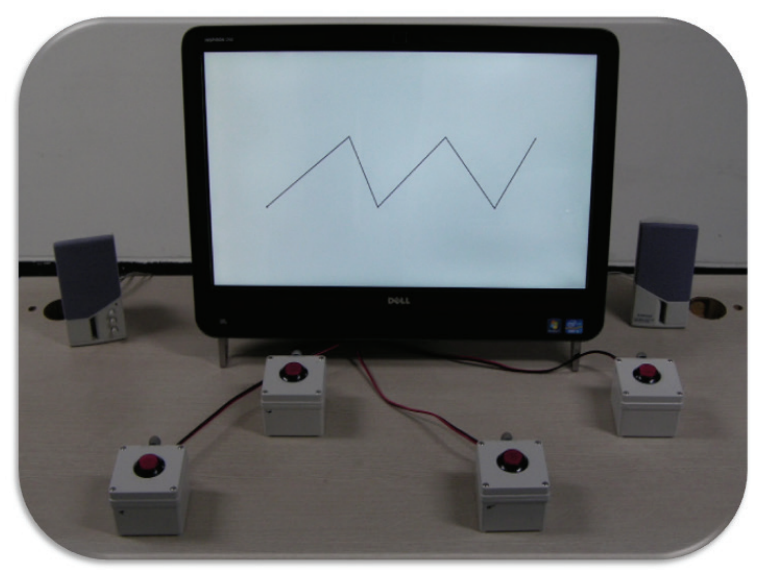

Fig. 1. Apparatus.

Table 1. Temporal structures of five segments of the goal rhythm pattern

\begin{tabular}{lccccc}
\hline Rhythm & Segment 1 & Segment 2 & Segment 3 & Segment 4 & Segment 5 \\
\hline A & $31.25 \%$ & $10.50 \%$ & $25.00 \%$ & $18.75 \%$ & $14.50 \%$ \\
B & $18.75 \%$ & $31.25 \%$ & $10.50 \%$ & $14.50 \%$ & $25.00 \%$ \\
C & $10.50 \%$ & $25.00 \%$ & $14.50 \%$ & $31.25 \%$ & $18.75 \%$ \\
\hline
\end{tabular}

ulus on the screen. Goal rhythm pattern is shown as Table 1.

The visual stimulus depicted consisted of five segments presented from left to right direction and the auditory stimulus in the form of 25 -msec beep length was presented by sound structure (doe tone, $523.25 \mathrm{~Hz}$; sew tone, $784 \mathrm{~Hz}$ ) (Fig. 1).

\section{Experimental procedure: experiment 1 (coordination pattern)}

All participants were informed preliminary how to perform the experimental task, they were randomly assigned into two groups, OHG $(n=20)$ and BHG $(n=20)$. All participants performed pretest (1 block of 6 trials) with only dominant hand in order to examine the differences of timing ability between the two groups prior to the experiment. In the pretest, the rhythm structure was same with the duration of total length $(3,600 \mathrm{msec})$, while the different rhythm structure used. In the acquisition and retention phase, OHG practiced with only dominant hand and BHG practiced with two-hands in the phases of pretest, acquisition and retention. Participants performed 48 trials ( 6 trials/block, 8 blocks) with rhythm structures A, B, and C on each group. Approximately $24 \mathrm{hr}$ after acquisition phase, they performed 6 trials in unilateral retention and bilateral retention tests, in which were the same conditions as experienced during the acquisition phase.

\section{Data analysis and reduction}

The dependent variables of interest were relative-timing and absolute-timing errors. Absolute-timing error, an index of parameter specification error, was computed as absolute-timing error $(\mathrm{msec})=\left(C E^{2}+V E^{2}\right)^{1 / 2}$, where $C E$ is a measure of response bias that is computed as the average of the signed total movement time errors, and $V E$ is a measure of response variability that is computed as the standard deviation of the signed errors.

Relative timing, an index of generalized motor program (GMP) errors, was computed as the sum of the absolute differences between the goal percentages and the actual percentages for each segment. In equation form, Relative-timing error $(\%)=\mid \mathrm{R}_{1}-$ $31.25|+| \mathrm{R}_{2}-10.50|+| \mathrm{R}_{3}-25.00|+| \mathrm{R}_{4}-18.75|+| \mathrm{R}_{5}-14.50 \mid$, where $\mathrm{Rn}=($ actual movement time $[\mathrm{MT}]$ of segment $\mathrm{n} /$ total MT $\times 100$ ) 
Thus, $\mathrm{R}_{1}-\mathrm{R}_{5}$ are the proportions of total movement time used in segments $1-5$, respectively.

\section{Statistical analysis}

In the pretest, comparing differences between OHG and BHG were analyzed using the independent sample $t$-test. The acquisition phase, relative-timing errors and absolute-timing errors in groups and blocks $(\mathrm{OHG}$ and $\mathrm{BHG} \times 8$ blocks) were analyzed by the two-way repeated measures analysis of variance (ANOVA) on the block. The retention test in two groups $(\mathrm{OHG}$ and $\mathrm{BHG}) \times 3$ tests (pretest, one-hand retention test, two-hands retention test) were conducted by paired $t$-test. The IBM SPSS Statistics ver. 20.0 (IBM Co., Armonk, NY, USA) was used with the level of significance set at $P<0.05$.

\section{Experimental procedure: experiment 2 (observation pattern task)}

All participants were informed preliminary how to perform the experimental task, they were randomly assigned into two groups, active observation group (AOG; $\mathrm{n}=20$ ) and passive observation group (POG; $n=20$ ). All participants performed pretest performed pretest (1 block of 6 trials) with only dominant hand in order to examine the differences of timing ability between two groups prior to the experiment. In the pretest, the rhythm structure was same with the duration of total length $(3,600 \mathrm{msec})$, while the different rhythm structure used. In the acquisition and retention phase, AOG practiced with two-hands in the phases of acquisition and retention and POG observed AOG's practicing in the phases of acquisition and retention. Participants performed 48 trials ( 6 trials/block, 8 blocks) with rhythm structures A, B, and $\mathrm{C}$ on each group. Approximately $24 \mathrm{hr}$ after acquisition phase, they performed 6 trials in one-hand retention and two-hands retention tests, in which were the same conditions as experienced during the acquisition phase.

\section{Data analysis and reduction}

The dependent variables of interest were relative-timing and absolute-timing errors. Absolute-timing error, an index of parameter specification error, was computed as absolute-timing error $(\mathrm{msec})=\left(C E^{2}+V E^{2}\right)^{1 / 2}$, where $C E$ is a measure of response bias that is computed as the average of the signed total movement time errors, and $V E$ is a measure of response variability that is computed as the standard deviation of the signed errors.

Relative timing, an index of GMP errors, was computed as the sum of the absolute differences between the goal percentages and the actual percentages for each segment. In equation form, Relative-timing error $(\%)=\left|\mathrm{R}_{1}-31.25\right|+\left|\mathrm{R}_{2}-10.50\right|+\left|\mathrm{R}_{3}-25.00\right|+\mid \mathrm{R}_{4}$ $18.75|+| \mathrm{R}_{5}-14.50 \mid$, where $\mathrm{Rn}=$ (actual MT of segment $\mathrm{n} /$ total $M T \times 100)$ Thus, $R_{1}-R_{5}$ are the proportions of total movement time used in segments $1-5$, respectively.

\section{Statistical analysis}

In the pretest, comparing differences between AOG and POG were analyzed using the independent sample $t$-test. The acquisition phase, relative-timing errors and absolute-timing errors in groups and blocks (OHG and $\mathrm{BHG} \times 8$ blocks) were analyzed by the two-way repeated measures ANOVA on the block. The retention test in two groups $(\mathrm{OHG}$ and $\mathrm{BHG}) \times 3$ tests (pretest, onehand retention test, two-hands retention test) were conducted by the repeated measures ANOVA on the test. The IBM SPSS Statistics ver. 20.0 (IBM Co., Armonk, NY, USA) was used with the level of significance set at $P<0.05$.

\section{RESULTS}

\section{Experiment 1 (coordination pattern) \\ Absolute timing error}

Pretest: The independent samples $t$-test revealed no significant differences between OHG and BHG: $t(38)=-0.371, P>0.05$. This result showed that there were no differences in absolute-timing error between the two groups.

Acquisition: We computed the mean of absolute-timing errors for the two groups (Fig. 2). Two groups $\times 8$ blocks were analyzed by the repeated measures ANOVA on the blocks. In acquisition phase, there were significant main effects for the blocks: $F(7,266)=$ 17.085, $P=0.000$, while there were no significant main effects for the two groups: $F(1,38)=0.555, P=0.461$, and interaction effect between the groups and blocks: $F(7,266)=1.690, P=0.112$.

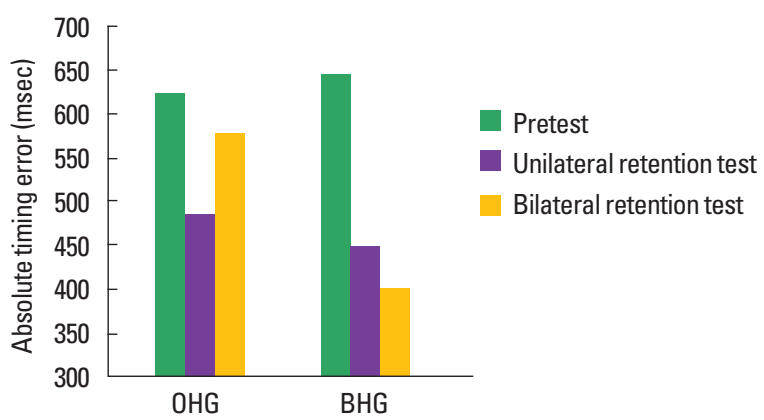

Fig. 2. Absolute timing error of two groups by coordination pattern in pretest, unilateral retention, and bilateral retention test. $\mathrm{OHG}$, one-hand practice group; $\mathrm{BHG}$, both-hands practice group. 
Retention: Absolute timing errors in retention tests were evaluated by the independent samples $t$-test. In OHG, the result indicated statistical significant differences in one-hand task: $t(19)=$ 3.142, $P=0.005$, while there was no statistical significant differences in two-hands task: $t(19)=1.035, P=0.314$. In BHG, the result indicated statistical significant differences not only in onehand task: $t(19)=5.315, P=0.000$, but also in two-hands task: $t(19)=3.504, P=0.002$.

\section{Relative timing error}

Pretest: The independent samples $t$-test revealed no significant differences between OHG and BHG: $t(38)=0.443, P>0.05$. This result showed that there were no differences in absolute-timing error between the two groups.

Acquisition: We computed the mean of relative-timing errors for the two groups (Fig. 3). Two groups $\times 8$ blocks were analyzed by the repeated measures ANOVA on the blocks. In acquisition phase, there were significant main effects for the blocks: $F(7$, 266) $=9.927, P=0.000$, while there were no significant main effects for the two groups: $F(1,38)=0.039, P=0.844$. There were

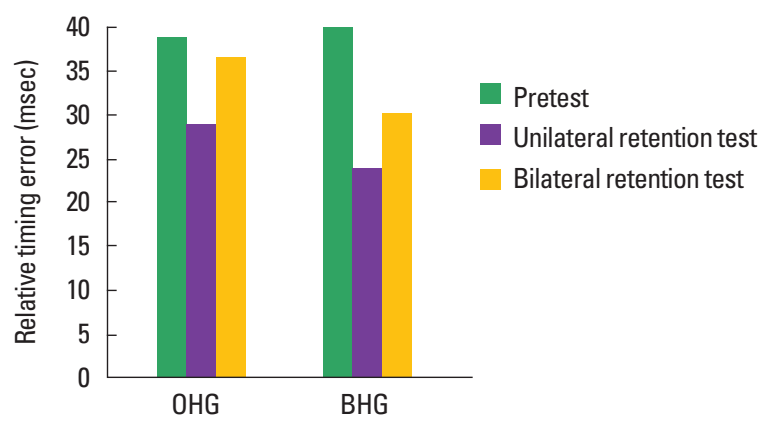

Fig. 3. Relative timing error of two groups by coordination pattern in pretest, unilateral retention, and bilateral retention test. $\mathrm{OHG}$, one-hand practice group; $\mathrm{BHG}$, both-hands practice group.

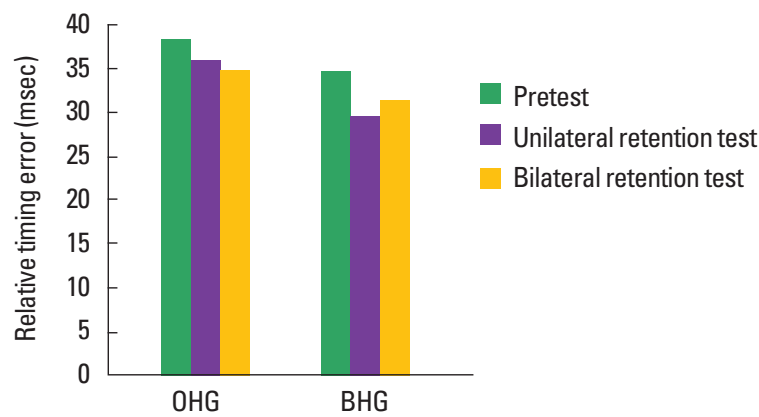

Fig. 4. Absolute timing error of two groups by observation pattern in pretest, unilateral retention, and bilateral retention test. $\mathrm{OHG}$, one-hand practice group; $\mathrm{BHG}$, both-hands practice group. significant interaction effect between the groups and blocks: $F(7$, 266) $=2.919, P=0.006$.

Retention: Relative timing errors in retention tests were evaluated by the independent samples $t$-test In OHG, the result indicated statistical significant differences in one-hand task: $t(19)=4.032, P=$ 0.001 , while there was statistical significant differences in two-hands task: $t(19)=0.881, P=0.389$. In BHG, the result indicated statistical significant differences not only in one-hand task: $t(19)=6.962, P=$ 0.000 , but also in two-hands task: $t(19)=2.999, P=0.007$.

\section{Experiment 2 (observation pattern task) Absolute timing error}

Pretest: The independent samples $t$-test revealed no significant differences between AOG and POG: $t(38)=0.181, P>0.05$. This result showed that there were no differences in absolute-timing error between the two groups.

Acquisition: We computed the mean of absolute-timing errors for the two groups (Fig. 4). Two groups $\times 8$ blocks were analyzed by the repeated measures ANOVA on the blocks. In acquisition phase, there were significant main effects for the blocks: $F(7$, $133)=3.744, P=0.001$, while there were no significant main effects for the two groups: $F(1,38)=0.675, P=0.371$, and interaction effect between the groups and blocks: $F(7,266)=1.589$, $P=0.102$.

Retention: Absolute timing errors in retention tests were evaluated by the independent samples $t$-test. In POG, the result indicated no statistical significant differences in one-hand task: $t(19)=0.714, P=0.484$, while there was statistical significant differences in two-hands task: $t(19)=2.560, P=0.019$. In AOG, the result indicated statistical significant differences not only in onehand task: $t(19)=2.312, P=0.032$, but also in two-hands task: $t(19)=2.358, P=0.029$.

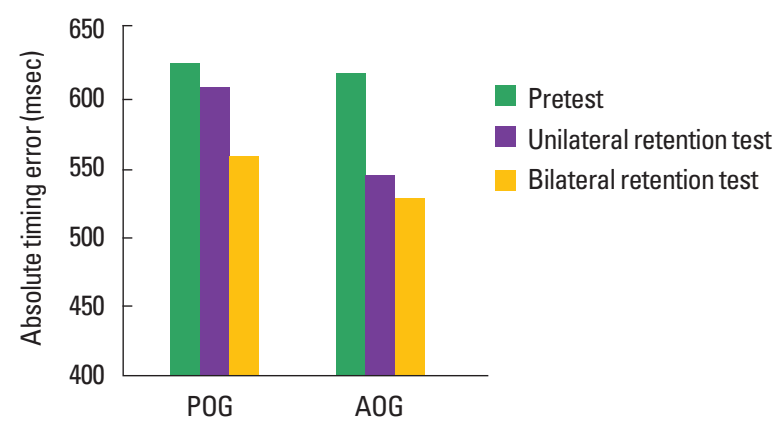

Fig. 5. Relative timing error of two groups by observation pattern in pretest, unilateral retention, and bilateral retention test. POG, passive observation group; $A 0 G$, active observation group. 


\section{Relative timing error}

Pretest: The independent samples $t$-test revealed no significant differences between AOG and POG: $t(38)=1.900, P>0.05$. This result showed that there were no differences in absolute-timing error between the two groups.

Acquisition: We computed the mean of relative-timing errors for the two groups (Fig. 5). Two groups $\times 8$ blocks were analyzed by the repeated measures ANOVA on the blocks. In acquisition phase, there were significant main effects for the blocks: $F(7$, $133)=4.363, P=0.000$, while there were no significant main effects for the two groups: $F(1,38)=0.042, P=0.734$. There were significant interaction effect between the groups and blocks: $F(7$, 266) $=2.719, P=0.009$.

Retention: Relative timing errors in retention tests were evaluated by the independent samples $t$-test. In POG, the result indicated no statistical significant differences in one-hand task: $t(19)=$ 2.013, $P=0.059$, while there was statistical significant differences in two-hands task: $t(19)=2.177, P=0.042$. In AOG, the result indicated statistical significant differences not only in one-hand task: $t(19)=4.379, P=0.000$, but also in two-hands task: $t(19)=$ $2.560, P=0.019$.

\section{DISCUSSION}

Studies of unilateral and bilateral exercises have been conducted as a kind of strategy to improve the affected upper-limb motor function in the field of remedial exercise. There have been conducted some studies on bimanual coordination and dominant hand according to skill level for the study of motor control and motor learning in normal subjects (Franz et al., 1996; Kim et al., 2012). However, it is hard to find a study focusing motor cognition in rehabilitation field. This study compared the learning efficacies of the OHG and BHG in needs of various changes of the upper-limb exercise types with MCI patients. Furthermore, this study investigated learning efficacy by observation types in active and passive pattern.

The efficacies of unilateral training and bilateral coordination training, activating the left and right cerebral hemispheres are stimulated differently by absolute timing and relative timing in the timing movement using the visual-auditory sensation information. Specifically, the both-hands practice was more effective than one-hand practice for absolute timing. This result goes with the previous study (Hallett, 2001) claiming that bilateral exercise is more effective than unilateral exercise, since it stimulates changes in the central and peripheral nervous system related to movements of the affected upper-limb. Even the neuromuscular junction is activated when both hands are coordinated. Therefore, bilateral coordination movement is thought to be more effective than unilateral coordination movement in terms of temporal-spatial timing perception.

Based on retention test, the BHG showed increased absolute timing ability compared to the OHG. In particular, the OHG showed increased absolute timing error values in each retention test compared to the preliminary test. This result reveals that the OHG perceived two-hands retention test as a new task for improving coordination. Bimanual coordination and temporal stability related studies (Ivry et al., 2002; Franz et al., 1996) show that the two-hands tapping is more efficient in terms of temporal and stability compared to the one-hand tapping, related to internal time signal. Thus, although one-hand movements only use the internal time signal of the relevant hand's direction, two-hands movements use the both internal time signals of each hand which enable performing its task in a stable manner. These results are hypothesized to be similar to such findings. Even though the time and rhythm structured tapping task is recognized with practice, it is perceived as a new task, due to changes in the use of the internal time signal. In general, AOG showed better learning effect than POG. One interesting thing is that there was a learning effect even in passive observation pattern. Such a result claimed for the elderly, who has problem to do daily activity, could use observation of temporal-spatial timing task for improving cognitive ability.

\section{CONFLICT OF INTEREST}

No potential conflict of interest relevant to this article was reported.

\section{ACKNOWLEDGMENTS}

This work was supported by the National Research Foundation of Korea Grant funded by the Korean Government (NRF-2014S1 A5B5A07042650).

\section{REFERENCES}

Dinse HR, Tegenthoff M, Heinisch C, Kalisch T. Ageing and touch. In: Goldstein EB, editor. The Sage encyclopedia of perception. London: Sage; 2009. p. 21-24.

Fernández-Ballesteros R, Zamarrón MD, Tárraga L, Moya R, Iñiguez J. Cognitive plasticity in healthy, mild cognitive impairment (MCI) sub- 
jects and Alzheimer's disease patients: a research project in Spain. Eur Psychol 2003;8:148-159.

Franz EA, Ivry RB, Helmuth LL. Reduced timing variability in patients with unilateral cerebellar lesions during bimanual movements. J Cogn Neurosci 1996;8:107-118.

Hallett M. Plasticity of the human motor cortex and recovery from stroke. Brain Res Brain Res Rev 2001;36:169-174.

Heyn P, Abreu BC, Ottenbacher KJ. The effects of exercise training on elderly persons with cognitive impairment and dementia: a meta-analysis. Arch Phys Med Rehabil 2004;85:1694-1704.

Ivry RB, Richardson TC, Helmuth LL. Improved temporal stability in multi-effector movements. J Exp Psychol: Hum Percept Perform 2002;
28:72-92.

Kim S, Roh M, Park S. Control of action on temporal timing across sensory information in different pitch structure. Korean J Sport Psychol 2012;23:213-225.

Maeda F, Kanai R, Shimojo S. Changing pitch induced visual motion illusion. Curr Biol 2004;14:R990-991.

Rapp S, Brenes G, Marsh AP. Memory enhancement training for older adults with mild cognitive impairment: a preliminary study. Aging Ment Health 2002;6:5-11.

Summers JJ, Kagerer FA, Garry MI, Hiraga CY, Loftus A, Cauraugh JH. Bilateral and unilateral movement training on upper limb function in chronic stroke patients: A TMS study. J Neurol Sci 2007;252:76-82. 